\title{
Neonatal Multisensory Processing in Preterm and Term Infants Predicts Sensory Reactivity and Internalizing Tendencies in Early Childhood
}

\author{
Nathalie L. Maitre ${ }^{1,2,12}$ (D) Alexandra P. Key ${ }^{2,3} \cdot$ James C. Slaughter $^{4} \cdot$ Paul J. Yoder $^{5}$ (D) Mary Lauren Neel ${ }^{1}$ (D) \\ Céline Richard ${ }^{1} \cdot$ Mark T. Wallace $^{2,3,6,7,8} \cdot$ Micah M. Murray $2,9,10,11$ (D)
}

Received: 16 June 2020 / Accepted: 13 July 2020 / Published online: 12 August 2020

(c) The Author(s) 2020

\begin{abstract}
Multisensory processes include the capacity to combine information from the different senses, often improving stimulus representations and behavior. The extent to which multisensory processes are an innate capacity or instead require experience with environmental stimuli remains debated. We addressed this knowledge gap by studying multisensory processes in prematurely born and full-term infants. We recorded 128-channel event-related potentials (ERPs) from a cohort of 55 full-term and 61 preterm neonates (at an equivalent gestational age) in response to auditory, somatosensory, and combined auditory-somatosensory multisensory stimuli. Data were analyzed within an electrical neuroimaging framework, involving unsupervised topographic clustering of the ERP data. Multisensory processing in full-term infants was characterized by a simple linear summation of responses to auditory and somatosensory stimuli alone, which furthermore shared common ERP topographic features. We refer to the ERP topography observed in full-term infants as "typical infantile processing" (TIP). In stark contrast, preterm infants exhibited non-linear responses and topographies less-often characterized by TIP; there were distinct patterns of ERP topographies to multisensory and summed unisensory conditions. We further observed that the better TIP characterized an infant's ERPs, independently of prematurity, the more typical was the score on the Infant/ Toddler Sensory Profile (ITSP) at 12 months of age and the less likely was the child to the show internalizing tendencies at 24 months of age. Collectively, these results highlight striking differences in the brain's responses to multisensory stimuli in children born prematurely; differences that relate to later sensory and internalizing functions.
\end{abstract}

Keywords Premature $\cdot$ Infant $\cdot$ Neonate brain $\cdot$ EEG $\cdot$ Multisensory $\cdot$ Touch $\cdot$ Sound $\cdot$ Hypersensitivity

\section{Introduction}

Despite decades of results on how information from the different senses is combined, we still know surprisingly little about how such multisensory processes develop in humans

Handling Editor: Christoph M. Michel.

Electronic supplementary material The online version of this article (https://doi.org/10.1007/s10548-020-00791-4) contains supplementary material, which is available to authorized users.

Nathalie L. Maitre

Nathalie.Maitre@nationwidechildrens.org

Micah M. Murray

Micah.Murray@chuv.ch

Extended author information available on the last page of the article
(Bremner et al. 2012; Murray et al. 2016). Nonetheless, it is recognized that the construction of a unified perceptual reality through multisensory interactions is essential for the establishment of concepts such as self vs. non-self, critical to social-emotional functioning (Aspell et al. 2012; Bahrick and Lickliter 2012; Lewkowicz 2014; Lickliter and Bahrick 2004; Rochat et al. 2012). During the neonatal period, when peripheral and central nervous systems are still developing, integration of lower-level physical stimulus features (such as duration, rhythm and intensity) is a first critical step in the maturation of multisensory processes (Murray et al. 2016). Theoretically, without the ability to integrate multiple streams of lower-level sensory attributes, an infant cannot interpret these stimuli and is left with perceiving the environment as confusing, distracting or even potentially hostile (Lewkowicz 2014; Lickliter 2011). 
There continues to be ongoing discussion and debate as to whether the development of multisensory processes is innate (though undoubtedly also tuned by environmental experiences) or instead arises after the child has accrued at least several months of experiences with the sensory world (Dionne-Dostie et al. 2015). While objective and quantitative studies in neonates and infants are challenging, a seminal study by (Lewkowicz and Turkewitz 1980) measured 3-4 week-old infants' heart rates and showed that these children can associate light and sound intensities. More recent results have shown that newborn infants can match numerosity across the senses (Izard et al. 2009) and that 4-month-old infants are sensitive to the spatial congruence of auditorytactile events (Thomas et al. 2018). However, one constraint of most studies in infants and young children is that they are typically based on child-appropriate behavioural measures, such as preferential looking, and consequently provide limited insights into the putative neurobiological mechanisms and maturation of multisensory processes. One pilot study, using MEG in a set of 9 infants as young as 6-months-old, demonstrated there to be non-linear auditory-somatosensory neural response interactions, defined as the difference in responses to simultaneous presentations of multisensory pairs versus the summed responses to auditory and somatosensory stimuli alone. Such responses were found to be more pronounced in 11-13 than in 6-9 month-olds (Stephen et al. 2007).

Thus, in light of the current knowledge gap concerning neonatal multisensory processes, the first objective of the present study was to record high-density EEG in neonates during auditory, tactile, and multisensory (i.e., audio-tactile) stimulus presentation and thus characterize the presence and core characteristics of multisensory neural response interactions.

The second objective of this study was to determine the role played by early-life experiences in shaping multisensory processes, contrasting cases of full-term and premature births. The nature of the sensory experiences received during the perinatal period can have a wideranging impact on later development across domains from sensory processing to higher cognitive function (Maitre et al. 2017; Nelson 2001; Sheridan and Nelson 2009; Thompson and Nelson 2000). In addition, the impact of sensory experiences undoubtedly extends into the gestational period, which likely has significant impact in cases when infants are born prematurely and spend all or some period of the final trimester in a neonatal intensive care unit (NICU) (Eckstein Grunau 2002; Foster and Verny 2007; Lickliter 2011). Of concern, therefore, is the fact that preterm infants are exposed for weeks or months to atypical sensory stimuli in the NICU (Santos et al. 2015). Preterm infants are exposed to few typical stimuli such as human voice, and there is some evidence that this may result in maturation of voice perception (Adam-Darque et al. 2020) at term equivalent age, as compared to infants born full-term. However, parental involvement in NICUs varies widely, and the majority of stimuli in NICUs neither approximate intrauterine nor home, infant-directed environments (Lahav and Skoe 2014; Oller et al. 2019; Best et al. 2018). After months cared for in the NICU, preterm speech sound discrimination and somatosensory processing appear to be worse than in term-born counterparts at discharge to home. Past research has also demonstrated that the effects of immaturity on sensory system development are not fully compensated in the first years (Brummelte et al. 2012; Key et al. 2012; Rose et al. 2008). Evidence for this comes from the diminished brain responses of preterm infants in response to sensory stimuli at discharge, with long-lasting developmental consequences (Key et al. 2012), including predicting worse developmental outcomes in toddlerhood (Maitre et al. 2013).

The third objective of this study was to then use an EEGbased measure of multisensory processes in neonates as a predictor of later outcome measures when these children were infants and toddlers (i.e., at 12 and 24 months, respectively). The consequences of preterm birth combined with atypical sensory experiences may result in behaviorallymeasured sensory processing problems, which are themselves later associated with concerns for behavioral adaptation and social emotional functioning (Cassiano et al. 2016). Infants with atypical sensory neurological thresholds to the home environment are challenged in their social and emotional interactions: they can require abnormally high levels of concurrent sensory stimuli to experience an interaction or they can become overwhelmed at low levels of stimulation diversity and intensity. These maladaptive responses are associated with worse behavioral outcomes in preterm and term infants (Machado et al. 2017). Early atypical neurobehaviors associated with behavioral sensory hypo- and hyper-responsiveness in later childhood can be detected at discharge from the hospital (Ryckman et al. 2017). Behavioral risk tendencies in early childhood are often precursors of behavioral disorders later in adolescence or adulthood, such as anxious, withdrawn, or fearful (internalizing) behaviors and aggressive, antisocial (externalizing) behaviors (Achenbach 2009). Preterm infants are at higher risk for internalizing and externalizing behaviors (Bhutta et al. 2002), and ensuing problems at adolescence and adulthood (Hack 2009; Hille et al. 2008; Turpin et al. 2019). This objective is also predicated on a growing literature in schoolchildren (Barutchu et al. 2011, 2019a, b, c; Birch and Belmont 1965; Denervaud et al. 2020; Rose et al. 2008, 2012) as well as older infants and toddlers showing that multisensory processes, in particular, are predictive of the integrity of various facets of cognitive functions, including memory, attention, executive functions, and fluid intelligence. 
In particular, the construction of auditory-tactile processing in infants and its later associations with atypical sensory thresholds and maladaptive behaviors such as excessive internalizing and external tendencies. Therefore, a knowledge gap in the development of multisensory processes exists, with little neurophysiological evidence regarding the construction of multisensory representations during the neonatal period, or how these representations are altered by early birth and the sensory environment experienced by the premature baby. Additionally, testing whether there are differences in the behavioral outcomes of infants with varying multisensory responses may suggest possible associations between later adaptations to sensory environments and behavioral tendencies similar to those studied in older children. Ultimately, understanding the construction of multisensory responses in infancy is necessary to rational design of optimal sensory environments and interventions addressing poor social-emotional and behavioral outcomes in childhood.

\section{Methods}

\section{Participants}

A cohort of healthy preterm and full-term infants was recruited from the newborn nursery and the Neonatal Intensive Care Unit at Vanderbilt University Medical Center (Nashville, TN, USA) (Table 1). These infants are the same as those previously reported (Maitre et al. 2017). The Institutional Review Board approved the study. Inclusion criteria were preterm infants $<37$ weeks gestational age (GA) at birth and full-term infants $\geq 37$ weeks GA. Exclusion criteria were lethal congenital abnormalities or severe abnormalities on any cranial imaging (cerebellar hemorrhage, intraventricular hemorrhage grade III or IV, periventricular leukomalacia, ischemia or stroke) or infants who had failed their auditory brainstem response testing performed at 34 weeks postmenstrual age (PMA).

\section{General Procedures and Variables}

\section{Overview}

Continuous EEG data were acquired using published protocols as near to discharge as possible in preterm infants (35-38 weeks PMA) and 1-3 days after birth for full-term infants. Primary caregivers completed the Infant/Toddler Sensory Profile (ITSP) (Dunn 2002) at 12-15 months corrected age (CA). The Child Behavior Checklist (CBCL) was completed at 24-27 months CA (Achenbach 2009; Dunn 2002). Primary caregivers received assistance from a trained therapist, as needed, to complete the assessments. In accordance with IRB regulations, all data were stored in a secure Research Electronic Data Capture (REDCap) encrypted server (Harris et al. 2019).

\section{EEG Acquisition and Pre-processing}

A high-density array net of 128 electrodes embedded in soft sponges (Geodesic Sensor Net, EGI, Inc., Eugene, OR, USA) continuously recorded the EEG using Net Station

Table 1 Participants' characteristics and outcomes

Demographics

\begin{tabular}{|c|c|c|c|}
\hline & All & Full-term $(\mathrm{N}=55)$ & Preterm $(\mathrm{N}=61)$ \\
\hline Gestational age, weeks, median (IQR) & $36(31,39)$ & $40(39,41)$ & $31(30,33)$ \\
\hline Sex $(\%$ female $)$ & 52 & 49 & 54 \\
\hline PND at testing, median (IQR) & $5(2,28)$ & $2(1,2)$ & $28(11,40)$ \\
\hline Maternal education (\% high school graduation) & 95 & 95 & 94 \\
\hline \multicolumn{4}{|l|}{ One-year ITSP outcomes } \\
\hline & All & Full-term $(\mathrm{N}=42)$ & Preterm $(N=49)$ \\
\hline Corrected age at testing, months, median (IQR) & $13(12,14)$ & $13(12,14)$ & $13(12,15)$ \\
\hline Atypical (\%) & 20 & 21 & 18 \\
\hline \multicolumn{4}{|l|}{ Two-year CBCL outcomes } \\
\hline & & Full-term $(\mathrm{N}=34)$ & Preterm $(N=44)$ \\
\hline Corrected age at testing, months, median (IQR) & $24(24,26)$ & $25(24,26)$ & $24(23,25)$ \\
\hline Internalizing: above risk threshold of $16(\%)$ & 65 & 55 & 72 \\
\hline Externalizing: above risk threshold of $20(\%)$ & 11 & 6 & 16 \\
\hline
\end{tabular}

$I Q R$ Interquartile range, $P N D$ postnatal days, ITSP Infant/Toddler Sensory Profile, $C B C L$ Child Behavior Checklist 
software (v. 4.3; EGI, Inc.) (Fig. 1). Data were sampled at $1000 \mathrm{~Hz}$. All infants were tested in his/her patient room while lying on their backs in the bassinet/crib or being held in the supine position by a caregiver. No restraint was used, and infants were tested in quiet alert states. ERP responses were collected in response to 50 randomly presented trials for each of four stimulus conditions. Intertrial intervals randomly varied between 2500 and $3000 \mathrm{~ms}$ between any trial, with no more than two events including a tactile stimulus presented in a row, to prevent tactile habituation (Maitre and Key 2014).

There were four stimulus conditions. The "tactile" condition was an air puff emanating from a nozzle that was positioned $5 \mathrm{~mm}$ above the skin of the palmar surface of

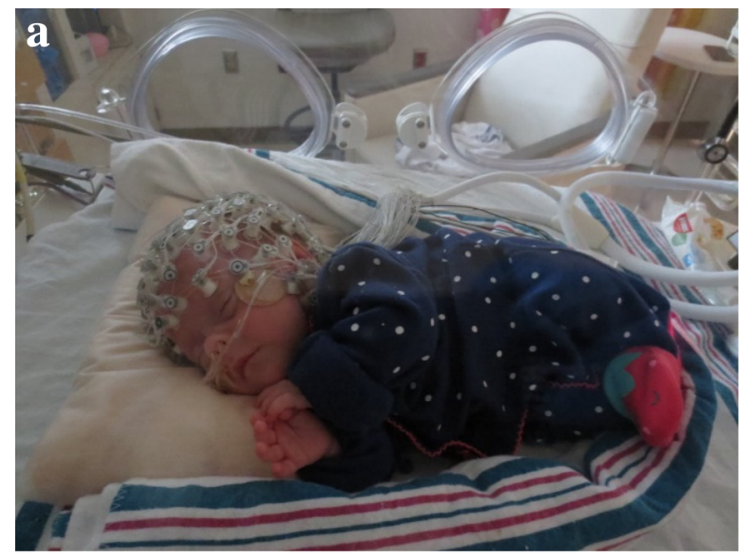

\section{c}
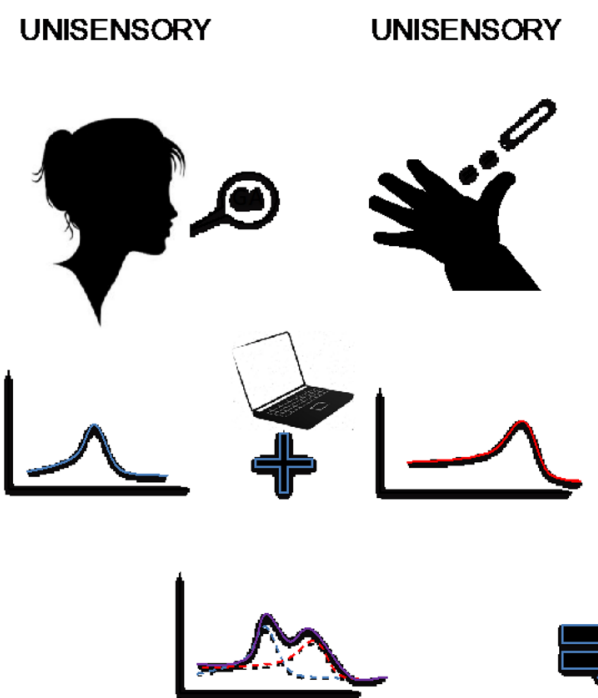

SUMMED UNISENSORY RESPONSES

Fig. 1 Neonates were tested using a 128-electrode EEG net in their isolettes a if they were in the NICU (preterm) or in their bassinettes in the newborn nursery (full-term). Calibrated tactile stimulation was delivered via a custom 3D-printed holder fastened with a neoprene glove to the infant's hand allowing the air pulse to always have the same pressure and target (b). The paradigm presents stimuli in ran- the right hand using a mold holder. The puff delivered a calibrated pressure of $5 \mathrm{psi}(34.5 \mathrm{kPa})$ over a $3 \mathrm{~mm}^{2}$ area, which approximates light touch. The device emitting the air puff also produced a mechanical sound as air exits the system through an alternate matching nozzle positioned away from the subject. The "sham puff" condition entailed only the mechanical sound of the device producing the air puff. That is, a second nozzle was pointed away from the infant's hand. This condition provided a control for the auditory stimulus that occurred when the air puff was delivered to the hand. The "auditory" condition involved presentation of a computer-synthesized /ga/ syllable from the "transition only" stimulus series employed by (Stevens and Blumstein 1978). The stimulus had no initial
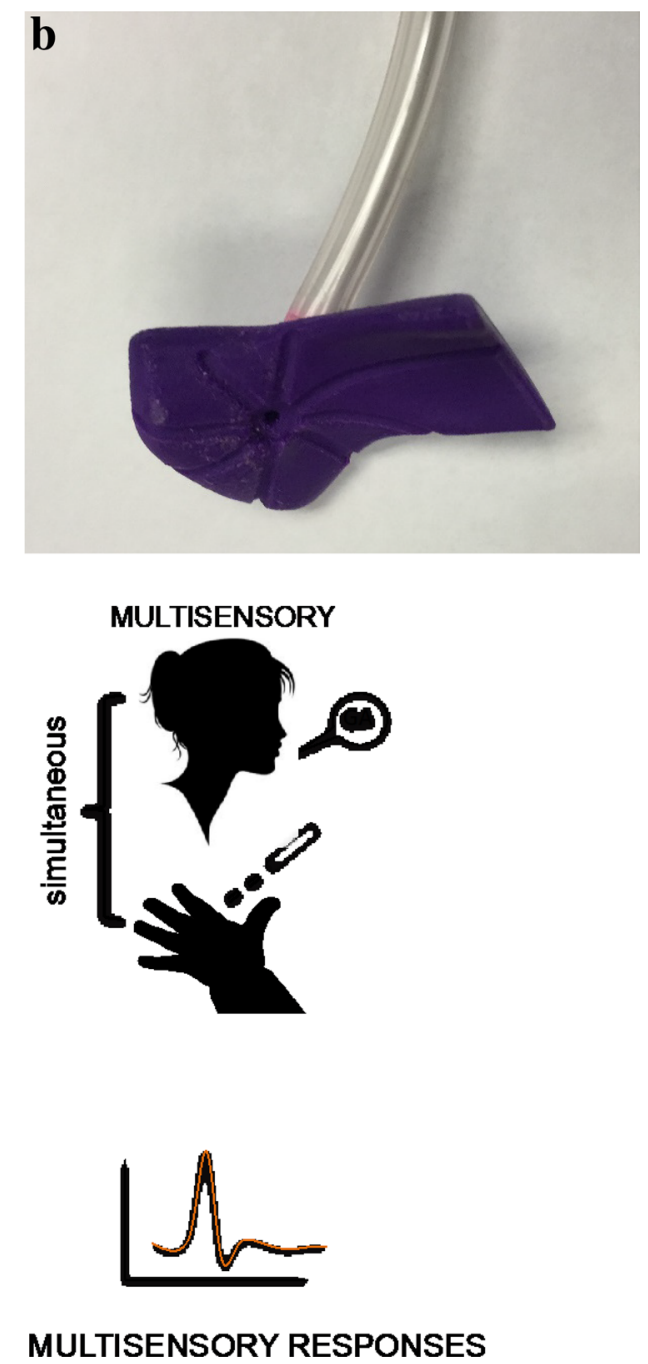

dom order and at random intertrial intervals, with a standard /ga/ sound and the tactile air puff stimulation presented separately for unisensory conditions or simultaneously for multisensory conditions (see c). Finally, in the analysis, the unisensory responses are algebraically added to produce a summed response and compared with the true multisensory response (panel c, lower) 
noise burst. The speech sound was synthesized on a Klatt (cascade) synthesizer so that the amplitude of the formant was modulated as a function of the respective formant frequencies, as in natural speech. Sound was delivered at 60 dBA using a speaker $12 \mathrm{~cm}$ at midline above the infant's head. The "sham puff" condition was delivered simultaneously and synchronously as the /ga/, allowing for the auditory condition to be compared with tactile and multisensory conditions. The "multisensory" condition was the simultaneous and synchronous delivery of the "tactile" and "auditory" conditions. Time-locking of simultaneous stimuli under $5 \mathrm{~ms}$ was confirmed using oscilloscopes as previously described (Maitre and Key 2014).

Data pre-processing and ERP analyses were implemented with the Cartool freeware (Brunet et al. 2011). Prior to epoching, the continuous EEG was filtered (lowpass $40 \mathrm{~Hz}$; high-pass $0.3 \mathrm{~Hz}$; using a second-order Butterworth filter with $12 \mathrm{~dB} /$ octave roll-off that was computed linearly in both forward and backward directions to eliminate phase shifts). Peri-stimulus epochs of continuous EEG spanned from $100 \mathrm{~ms}$ pre-stimulus onset to $500 \mathrm{~ms}$ post-stimulus onset. Artifact-contaminated epochs were identified via automated routines in Net Station and confirmed with visual inspection for movement, eye blinks and eye movements, as well as other sources of transient noise. An infant was deemed to have analyzable ERP data whenever there were more than 10 usable trials per each condition, with every usable trial also having more than 108 of 128 electrodes with artifact-free signals. Mean numbers of usable trials per conditions were however greater than the pre-specified minimum (multisensory condition $22.3 \pm 8.6$; tactile conditions $23.2 \pm 8.8$; auditory $22.8 \pm 8.9$ ). ERPs were the result of signal averaging epochs for each condition. Data at artifact-contaminated electrodes in the ERP were interpolated using 3D splines (Perrin et al. 1987). ERP data were then baseline-corrected using the $100 \mathrm{~ms}$ pre-stimulus interval and re-referenced to the common average reference.

ITSP The ITSP consists of a 48-item questionnaire assessing how and how often the child responds to different sensory experiences in the home environment (Dunn 2002). One key variable derived from this instrument is the infant's sensory reactivity, which is defined as atypical if it was either lower or higher than a standard range. 'Lower than typical' reflects an infant for whom minimal sensory input causes the infant to respond and 'higher than typical' reflects an infant for whom large amounts of stimulation are required before the infant responds (Dunn 2002). Typical and atypical ranges are provided for scores in the 7-12-month age band using published norms, generating a dichotomous variable. Using these norms, we classified infants into typical or atypical sensory reactivity subgroups.
CBCL Scores were obtained for internalizing and externalizing tendencies on the CBCL and risk group member was assigned using the Achenbach System of Empirically Based Assessment (ASEBA) manual (Achenbach and Rescorla 2000). Internalizing tendencies include anxiety, depression, withdrawn, and somatic complains. Using the guidelines accompanying the instrument, a cut-off of 16 on the internalizing items was used to classify infants into an "internalizer" subgroup or not. Externalizing tendencies include rule-breaking and aggressive behaviors. A cut-off of 20 on the externalizing items was used to classify infants into an "externalizer" subgroup or not. Internalizing and externalizing problems in early childhood are associated with specific psychopathology in adolescence and adulthood (Achenbach 2009; Hofstra et al. 2018, 2002).

\section{Results}

Of 116 infants tested in the NICU and newborn nursery, $78.5 \%$ were followed to 12 months $\mathrm{CA}$ and $68.1 \%$ were followed to 24 months CA. Full-term and preterm infants had similar rates of atypical sensory threshold in the home (Table 1).

\section{ERP Results}

Our first research aim was to ascertain whether infants exhibit non-linear neural response interactions to auditorytactile multisensory stimuli and, if so, to determine if this followed from modulations in response strength and/or response topography; the latter of which would be indicative of changes in the configuration of the underlying brain sources (reviewed in Biasiucci et al. 2019; Tivadar and Murray 2018). To achieve this, we compared ERPs to multisensory stimulus pairs with summed ERPs to the corresponding unisensory conditions (see Foxe et al. 2000; Murray et al. 2005; Sperdin 2009) for similar analyses of auditory-somatosensory interactions in adults). Specifically, we ran two sets of paired $t$-tests-i.e. one per group-comparing multisensory pair and summed unisensory ERPs at each scalp site as a function of time (Fig. 2). We then compared global field power, a measure of response strength, in a similar way and using a paired $t$-test as a function of time. Finally, we tested for topographic differences using global dissimilarity and a non-parametric permutation test. In all analyses, effects were considered reliable if significant differences were observed for a minimum of $20 \mathrm{~ms}$ contiguously (Guthrie and Buchwald 1991; Murray et al. 2018). For full-term infants there was no evidence of neural response differences between paired multisensory and summed unisensory conditions in either global field power or global dissimilarity. By contrast, for preterm infants, there were robust topographic 


\section{a Full-term infants}
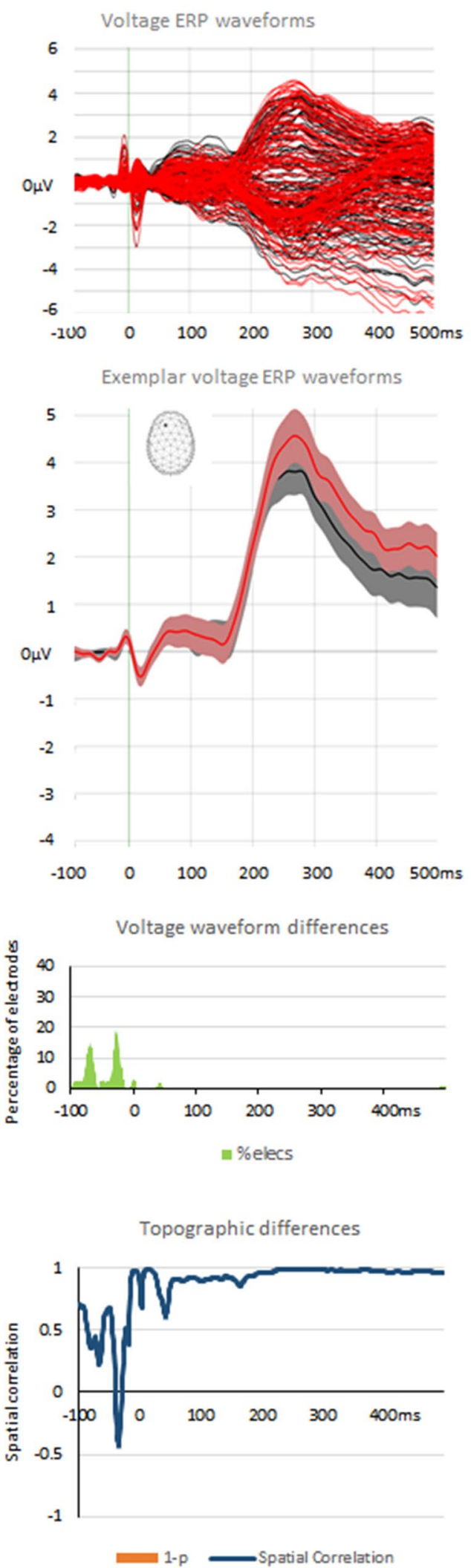

\section{b Preterm infants}

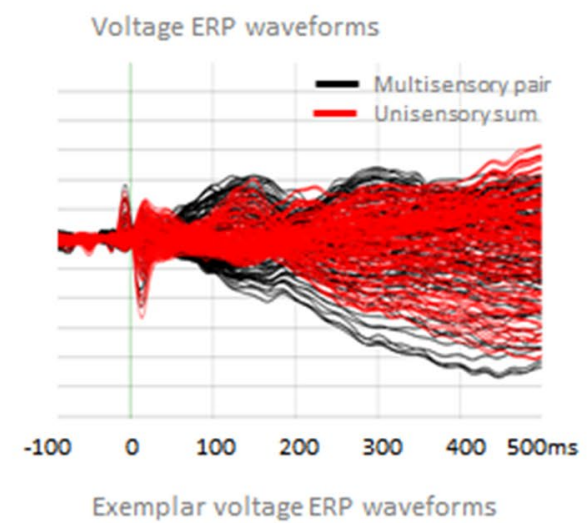

$\mathrm{OuV}$

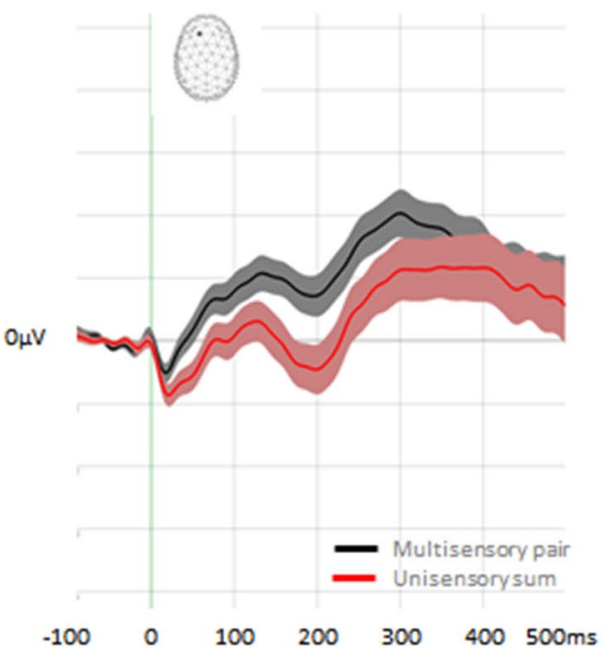

Voltage waveform differences

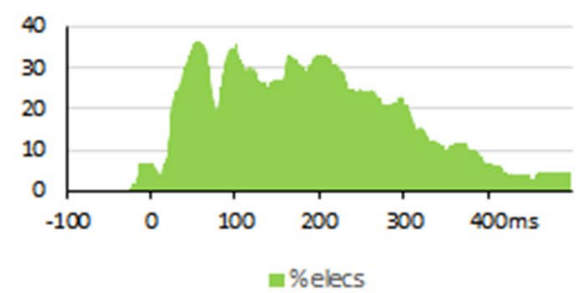

Topographic differences

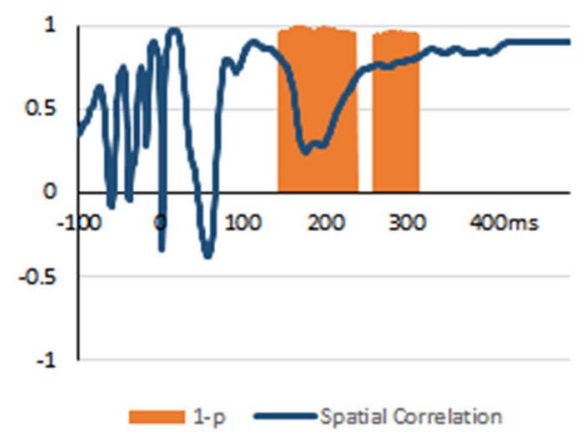


४Fig. 2 ERP analyses contrasting multisensory and summed unisensory responses from full term and preterm infants (panels $\mathbf{a}$ and $\mathbf{b}$, respectively). The uppermost row displays group-averaged data from all electrodes for the multisensory pair and summed unisensory conditions. The next row displays group-averaged data (s.e.m. indicated by shaded area) from an exemplar fronto-central electrode (see inset). The next row displays the percentage of the electrode montage exhibiting a significant difference as a function of time. The bottom row displays the spatial correlation between multisensory and summed unisensory responses (blue line) as well as time periods of significant differences (orange areas)

differences between multisensory pair and summed unisensory conditions during the $143-240 \mathrm{~ms}$ post-stimulus time period as well as $258-315 \mathrm{~ms}$ post-stimulus time period. There was no evidence of sustained modulations in global field power between paired vs. summed conditions in the preterm infants. We would also note that there were also differences at the level of voltage ERP waveforms beginning at approximately $40 \mathrm{~ms}$ post-stimulus onset, which is generally consistent in their timing with effects in adults (Murray 2005) and children (Brett-Green et al. 2008). However, we would remind readers that analyses of voltage ERP waveforms are reference-dependent and that the presence, timing and spatial distribution of significant differences will vary with the reference choice (cf. Murray et al. 2008; Tzovara et al. 2012; Tivadar and Murray 2018 for discussion). We therefore focus our interpetations on reference-independent measures; i.e. global field power and dissimilarity. This collective pattern would indicate that for preterm infants there are distinct brain networks responding to multisensory versus unisensory stimuli, whereas there was no evidence for such differences in full-term infants.

Our next research aim was to ascertain if and when temporally stable networks of brain activity engaged by infants differ as a function of preterm status and/or stimulus condition. To address this research aim, we used a multistep process. First, we applied an unsupervised hierarchical cluster analysis to the group-averaged (regardless of preterm status) ERP data that was concatenated across responses to the multisensory and summed unisensory tactile and auditory conditions. This clustering identified the minimal number of microstates, which are represented as temporally stable patterns of brain activity at the scalp (hereafter template maps), to account for the maximal global explained variance in the entire ERP dataset. In the present case, 16 clusters that involved 12 different template maps yielded a global explained variance of 96.3\% (see Supplementary Fig. 1).

Second, after re-ascribing preterm status as well as multisensory and summed unisensory condition labels back onto the template maps, it is possible to generate data-driven hypotheses regarding time windows when different template maps appear to better characterize either preterm status and/ or condition. There were two time windows during which the microstates characterizing the group-averaged data appeared different: 176-231 and 232-375 ms. During each of these time windows, three different template maps primarily characterized the brain responses in the group-averaged data. These were labeled Maps A-C for the earlier time window and Maps D-F for the later time window (Fig. 3 and Supplementary Fig. 2, respectively).

Third, for each time window separately, we calculated the spatial correlation between each template map and each infant's ERP data from the multisensory and summed unisensory conditions. These spatial correlations indicate for each time frame which of the three template maps best matches the brain response from each participant and each condition. From these within-participant spatial correlations, we computed the proportion of a temporal window characterized by each template map by each condition.

Fourth, these proportions were then submitted to a mixedmodel ANOVA using Preterm Status (preterm vs. full-term) as the between-subjects factor and both Condition (multisensory vs. paired unisensory) and Map (either Maps A-C or Maps D-F, depending on the time window analyzed) as the within-subjects factors. This analysis allowed us to determine if there were topographic differences between conditions (by extension differences in active brain networks) that varied as a function of preterm status. It also allowed us to quantify the degree to which topographic patterns in the preterm infants resembled those observed in full-term infants. Presumably, the pattern and preponderance of template maps during stimulus processing in full-term infants is more adaptive than those observed in preterm infants [see also (Maitre et al. 2017) for the case of brain responses to light touch].

For the earlier time window (176-231 ms), Maps A-C were fitted to the data (Fig. 3). The ANOVA revealed a significant main effect of Map $\left(\mathrm{F}_{(1.67,190.45)}=17.96 ; p<0.001\right.$; $\left.\eta_{p}{ }^{2}=0.14\right)$, a significant Condition $\times$ Map interaction $\left(\mathrm{F}_{(1.86,211.71)}=4.59 ; p=0.013 ; \eta_{p}{ }^{2}=0.04\right)$, as well as a significant Preterm Status $\times$ Map interaction $\left(\mathrm{F}_{(1.67,190.45)}=12.72\right.$; $\left.p<0.001 ; \eta_{p}{ }^{2}=0.10\right)$. The 3 -way interaction was not significant $(\mathrm{F}<1)$. In light of the Condition $\times$ Map interaction, indicating that each condition was better characterized by maps to varying degrees as well as the absence of a significant 3-way interaction, we next performed 1-way ANOVAs for each condition separately and collapsing across Preterm Status. For the multisensory pair condition, there was an effect of Map $\left(\mathrm{F}_{(1.87,215.54)}=7.94 ; p<0.001 ; \eta_{p}{ }^{2}=0.07\right)$. Map A predominantly characterized responses to multisensory stimulus pairs. On average, Map A best correlated with individual infant's brain responses $47.7 \pm 4.2 \%$ of the time (mean \pm s.e.m.), which was significantly more than either Map B $\left(22.3 \pm 3.3 \% ; \mathrm{t}_{(115)}=3.91 ; p<0.001\right)$ or Map $\mathrm{C}\left(30.0 \pm 3.8 \% ; \mathrm{t}_{(115)}=2.43 ; \mathrm{p}<0.02\right)$; the latter two of which did not differ $(\mathrm{p}>0.18)$. For the summed unisensory condition, there was an effect of Map $\left(\mathrm{F}_{(1.58,181.24)}=15.58\right.$; 
Template maps and single-subject fitting

(176-231ms post-stimulus period)

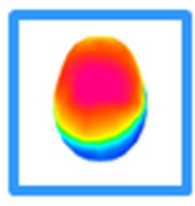

MapA

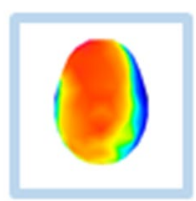

MapB

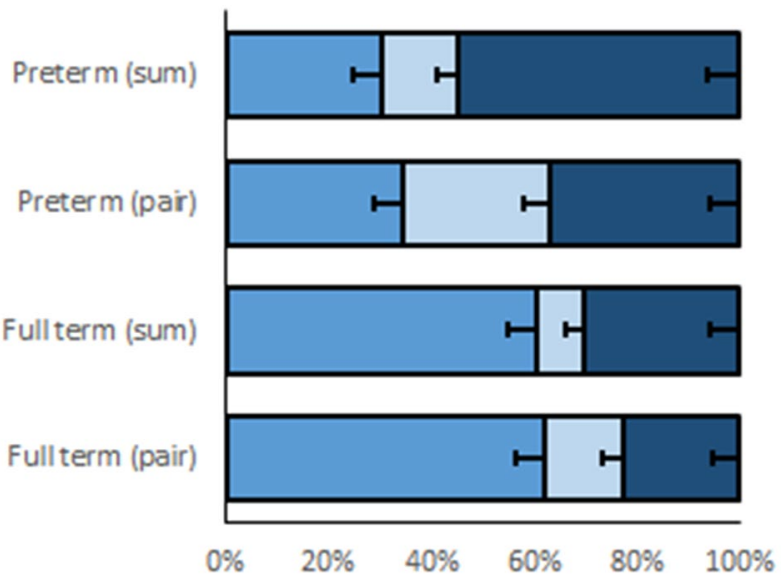

Fig. 3 Template maps identified over the 176-231 ms post-stimulus period via unsupervised hierarchical clustering of the ERP topography using the group-averaged dataset concatenated across conditions and full-term and preterm infants. The maps are displayed with the nose upwards and left hemiscalp on the left. The bar graphs display the single-subject fitting based on the spatial correlation between the template maps shown the top row and each time point of the infant's data over the 176-231 ms post-stimulus period. The bars show the average percentage of time each template map yielded the highest spatial correlation (error bars indicate s.e.m.) $\left.p<0.001 ; \eta_{p}{ }^{2}=0.12\right)$. Map A and Map C characterized responses $44.8 \pm 4.3 \%$ and $43.1 \pm 4.3 \%$ of the time, respectively, with no difference in these percentages $(p>0.84)$. By contrast, Map B characterized responses $12.1 \pm 2.7 \%$ of the time, which was significantly less than either Map A $\left(\mathrm{t}_{(115)}=5.71 ; \mathrm{p}<0.001\right)$ or Map C $\left(\mathrm{t}_{(115)}=5.51 ; p<0.001\right)$. Given this pattern of results, we considered the percentage of time responses were characterized by Map A as representative of typical infantile processing; "TIP".

We next assessed how TIP was associated with behavioural outcomes. First, the degree of prematurity at birth (indexed by GA) was a reliable predictor of the how often Map A characterized stimulus processing over the 176-231 ms post-stimulus time window ( $p<0.001$ for all multivariable regression analyses). For example, the probability of an infant born at 25 weeks GA of having ERP responses predominantly characterized by Map A is 27\%, whereas it rises in a linear fashion to $60 \%$ for an infant born at 40 weeks GA. Noting that Map A not only characterized responses to multisensory stimuli, but also to the summed unisensory condition, we examined this correlation when controlling for the percentage of time Map A characterized an infant's response to the summed unisensory condition. It was no longer associated (partial $r=0.09, p=0.5$ ).

We next assessed the extent to which stimulus processing near birth, as indexed by the relative preponderance of Map A in characterizing ERP responses to the multisensory stimulus over the 176-231 ms post-stimulus time window, was predictive of the typicality of sensory reactivity in the home measured at 12 months of age with the ITSP. This was indeed the case regardless of whether GA or postnatal days (PND) of test was statistically controlled (Table 2). To further assess the specificity of the association, we examined the same association after statistically controlling the relative preponderance of Map A in characterizing ERP

Table 2 Associations between EEG multisensory measures and behavioral outcomes

\begin{tabular}{llll}
\hline & Unadjusted OR $^{\mathrm{a}}[\mathrm{CI}]$ & Adjusted for GA OR ${ }^{\mathrm{a}}[\mathrm{CI}]$ & Adjusted for PND OR ${ }^{\mathrm{a}}[\mathrm{CI}]$ \\
\hline $\begin{array}{l}\text { Odds ratio of typicality of sensory reactivity at } 12 \text { months of } \\
\text { age }\end{array}$ & $0.26[0.07-0.96]^{*}$ & $0.23[0.06-0.86]^{*}$ & $0.21[0.06-0.80]^{*}$ \\
$\begin{array}{l}\text { Odds ratio for internalizing tendencies above threshold at } \\
\quad 24 \text { months of age }\end{array}$ & $0.22[0.06-0.83]^{*}$ & $0.24[0.06-0.91]^{*}$ & $0.25[0.07-0.93]^{*}$ \\
$\begin{array}{l}\text { Odds ratio for externalizing tendencies above threshold at } \\
24 \text { months of age }\end{array}$ & $0.50[0.1-2.54]$ & $0.51[0.09-2.72]$ & $0.49[0.09-2.57]$ \\
\hline
\end{tabular}

The top row shows unadjusted and adjusted odds ratio of typicality of sensory reactivity at 12 months of age as a function of the preponderance of Map A characterizing brain activity in response to multisensory stimuli. The lower two rows show unadjusted and adjusted odds ratio of internalizing and externalizing group membership at two years as a function of the preponderance of Map A characterizing brain activity in response to multisensory stimuli

GA gestational age at birth, $P N D$ postnatal days

${ }^{a}$ Odds ratios (OR) less than 1 indicate that children have a greater probability of having typical sensory reactivity, typical internalizing tendencies, and typical externalizing tendencies

$* p<0.05$ 
responses to the summed unisensory condition. The partial correlation was significant (partial $r=-0.3, p=0.04$ ).

Finally, we assessed whether stimulus processing near birth, again indexed by the relative preponderance of Map A in characterizing ERP responses over the 176-231 ms post-stimulus time window during the multisensory condition, was predictive of internalizing and externalizing group membership at 24 months of age as measured by the CBCL. Infants whose brain activity was more often characterized by Map A had lower odds of having internalizing scores above risk thresholds, regardless of whether GA or PND was statistically controlled. To examine whether this association was specific to the multisensory condition, we tested the association after controlling for the relative preponderance of Map A in characterizing ERP responses over the 176-231 ms post-stimulus time window during the summed unisensory condition. This association was significant, partial $r=-0.39, p=0.006$. There was no similar evidence in the case of externalizing tendencies (Table 2).

\section{Discussion}

We provide the first electrophysiologic characterization of auditory-somatosensory multisensory processing in fullterm and preterm neonates at their discharge from the hospital. Multisensory processing in full-term neonates was characterized by a linear addition of unisensory signals and by the preponderance of a single ERP topography for responses both to multisensory stimulus pairs and summed responses to unisensory conditions-what we refer to as "typical infantile processing" or TIP. By contrast, multisensory processing in preterm infants was characterized by non-linear neural response interactions and less frequent TIP, with responses more often characterized by additional ERP topographies. We further showed that multisensory processes, as indexed by TIP, were a reliable predictor of typical sensory thresholds when the child, independently of preterm status, was aged 12 months, as well as the absence of internalizing behavioral tendencies at 24 months. This was the case even after controlling for contributions of GA, postnatal age, and unisensory processes. This overall pattern of results highlights the importance of the establishment of the neural networks that support typical multisensory processes during early life and how the integrity of these networks and processes may foretell later sensory and clinical measures.

Together, these data provide evidence supporting the construct validity of topographic ERP analyses as a measure of the typicality of multisensory processing in neonates. Additionally, they support the hypothesis that one reason children with preterm birth tend to experience more internalizing symptoms may be at least partially grounded in the fact that they process multisensory stimuli differently as compared with their full-term peers. To understand the implications of our findings, it is useful to place them into the greater context of what is known about multisensory integration in older children, as no other study has examined brain-based measures in response to multisensory stimuli this early in development.

\section{Multisensory Processes Across the Lifespan}

The first set of results in our study centers on multisensory interactions in response to the combination of audition and touch. While full-term infants exhibited a linear response to such combinations, such that there were no differences between the responses to multisensory and summed unisensory conditions, preterm infants exhibited non-linear neural response interactions that began at approximately $145 \mathrm{~ms}$ post-stimulus onset. Moreover, this non-linearity was the result of topographic differences between responses to multisensory and summed unisensory conditions (see Figs. 2 and 3 ). These findings need to be considered against the backdrop of findings in older infants, aged 6-12 months (Stephen et al. 2007), older children, aged 6-13 years (Brett-Green et al. 2008; Russo et al. 2010), as well as adults (Foxe et al. 2000; Murray et al. 2005). In a small pilot study of 9 infants, (Stephen et al. 2007) claimed that non-linear response interactions were larger in the subset of four 11-13 month-olds than in five 6-9 month-olds, suggesting a developmental increase in these non-linear effects. Although there was no explicit reporting of the timing or topography of these effects, visual inspection of their data suggests that these effects are present during the initial $100-150$ ms post-stimulus onset in both age groups (cf. their Figs. 1 and 2). These data appear consistent with data that has been obtained from older children, who exhibit non-linear neural response interactions within the first $100-150 \mathrm{~ms}$ post-stimulus onset (Brett-Green et al. 2008). Such effects appear accelerated in adults, in which these interactions are seen within the first 50-90 ms post-stimulus onset (Murray et al. 2005). Collectively, these results suggest that non-linear auditorysomatosensory neural response interactions emerge within the first year of life. The present results build on this literature by showing that non-linear neural response interactions are not observable in full-term neonates at the time of their discharge from the hospital. Robust ERPs were observable in response to stimuli from each sensory modality alone, and the response to multisensory stimulus pairs was equivalent to the summed responses to each constituent stimulus alone. In these full-term neonates, there was no evidence for non-linear neural response interactions nor evidence for topographic differences between responses to multisensory pairs and summed unisensory conditions. One strong implication of these results is that early-life experience is likely to 
be a major contributor to the emergence of non-linear neural response interactions as neonates transition into infancy.

Our observation of robust non-linear neural response interactions in our group of preterm neonates who were recorded at an equivalent GA as the full-term neonates, lends support to the idea of early-life experience as a major contributor to the development of multisensory processes. As detailed above, the sensory experiences of preterm neonates dramatically differ from those of full-term neonates. Studies in developing animals have shown how strongly early-life experiences can shape patterns of multisensory integration. For example, animals reared under conditions where multisensory events were temporally synchronous but spatially separated results in robust multisensory integration to these pairings - a finding strikingly different from circumstances in which animals are reared with temporally and spatially contiguous stimuli events (Wallace and Stein 2007).

In addition to the contributions of atypical early-life experiences, it must also be acknowledged that the preterm brain is more immature at birth when compared with the fullterm brain. We therefore cannot readily disentangle whether preterm infants, at the time of discharge from hospital, are prone to manifest multisensory processing because they are now on a distinct developmental trajectory and/or because they are exhibiting accelerated maturation of an otherwise intact process. However, two aspects of the results are worth noting. First, the multisensory processes we observed in the preterm group were the result of topographic differences between responses to multisensory and summed unisensory conditions, which differs from the findings in adults in which multisensory interactions are driven almost exclusively by differences in response strength (Murray et al. 2005; Sperdin 2009). That is, auditory-somatosensory multisensory interactions in adults are characterized by the responses of a common brain network, whereas in preterm infants these processes appear dependent upon the activity within multiple networks. Comparable topographic analyses have not been conducted to date or reported in children. Second, the extent to which the topography of a preterm neonate's brain responses were characterized by the standard topography, what we refer to as TIP, directly related to the degree of prematurity. This would suggest that brain maturation itself contributes to the patterns of multisensory processes we have observed. Given the immaturity of these preterm infants' brains and coupled with their altered sensory environments, we propose that the development of the multisensory processes observed here are likely indicative of a distinct trajectory rather than simply an acceleration of what is otherwise neurotypical. However, we would hasten to add that additional data, including in utero functional data, would be required to support or refute this proposition with any level of conviction. However, there is now mounting quantitative investigation of the altered sensory experience in the NICU versus home environment (Liszka et al. 2020) as well as of the potential benefical effects of sensory enrichment (Webb et al. 2015). Nonetheless, our proposition that the multisensory responses we observed are the result of immature brain circuits and atypical sensory experience receives some measure of support in the set of correlational analyses that establish links between brain function and later outcome measures.

\section{Associations Between Multisensory Processes and Later Developmental Outcomes}

The second set of results concerns how multisensory brain responses, as characterized by their topography and more specifically by TIP, are predictive of later sensory thresholds and internalizing tendencies. These results were obtained across the cohort of both full-term and preterm infants. Thus, this brain-based functional index of multisensory processing appears to be particularly informative about the later integrity of other neurodevelopmental outcomes. This is in agreement with a growing body of evidence showing that multisensory processes may be particularly informative about the integrity of higher-level functions, and likely scaffold the development of these higher-order abilities. For example, Denervaud et al. (2020) showed in schoolchildren that multisensory gains on a simple detection task are predictive of both working memory and fluid intelligence measures, even when controlling for age. Likewise, Murray et al. (2018) used a similar multisensory detection task to accurately classify healthy ageing from older individuals with mild cognitive impairment. In these studies at opposite ends of the lifespan, the effects were specific for multisensory processes; unisensory processes were ineffective predictors of these cognitive functions. In the current work we extend these links between functional multisensory measures to later developmental indices that measure sensory thresholds at one age (12 months) and internalizing tendencies at a later age (24 months).

It must be pointed out here that correlational analyses cannot exclude the possibility that additional variables might also explain the associations of interest. Consequently, we cannot draw inference regarding causality here, even if implied by the longitudinal design. Nonetheless, our study's findings are consistent with a hypothesis that atypical multisensory processing may contribute to greater internalizing symptoms 24 months of age; a plausible view given that the multisensory stimulation received in early life (in an environment such as a NICU) may be a negative experience for some newborns. Because our cohort includes a large proportion of preterm infants, most atypical behavioral tendencies at 2 years of age are on the internalizing or dysregulated end of the spectrum instead of the externalizing one, consistent with published literature (Spittle et al. 2009). Internalizing 
tendencies are often indicative of withdrawal or fearful behaviors, with both passive and active hypersensitivity reactions. These concepts overlap with sensory processing frameworks that describe children's neurological threshold to environmental stimuli in their home or school environment: in these models, infants with low neurological thresholds to sensory stimuli can adapt to their threshold using active strategies such as avoiding (withdrawing from them) or passive sensitivity. However, the hypothesis that construction of multisensory responses in infancy contributes to atypical sensory reactivity and later internalizing tendencies can be better assessed using an experimental research design that manipulates multisensory processing and follows the infants for 24 months to assess internalizing symptoms.

\section{Limitations}

This study's main limitations include its correlational nature and size. Although the largest study of its kind, it was not designed to investigate causality or maturation, which would require longitudinal follow-up using consistent EEG paradigms throughout. Additionally, follow-up of the cohort to 2 years of age proved challenging in a large rural area $\left(>100,000 \mathrm{~km}^{2}\right)$ with high population mobility and highly variable health-insurance status influencing access to clinically-indicated follow-up services. Approximately $10 \%$ of Tennesseans had no health insurance in 2018, and about half the population receives job-based coverage through an employer instead of federally-provided insurance (Pellegrin 2020). Therefore, future confirmatory research is needed to determine the replicability of these findings.

\section{Conclusion}

We showed that the integrity of sensory and multisensory processes in full-term and preterm neonates can be quantitatively studied using high-density EEG and topographic analyses, showcasing the versatility of EEG techniques (Biasiucci et al. 2019). Future studies could use the proposed measure of typicality of multisensory processes (TIP) to determine whether interventions targeting multisensory processes affect functional outcomes, through improved typicality of the brain's response to (multisensory) stimuli. Still, this remains the first study to offer evidence for the very early emergence of multisensory processes in human infants, whether born full-term or preterm, and their association with later sensory reactivity and behavioral problems throughout the lifespan.

Acknowledgements Open access funding provided by University of Lausanne. This work has been supported by the National Institutes of
Health (USA) (1 K23 HD074736-01A1; 01/14-01/15 to NLM) and the Swiss National Science Foundation (Grant 169206 to MMM).

Author Contributions NLM conceived and designed the study, acquired and analyzed the data, drafted the manuscript created the figures, and obtained funding. APK helped conceptualize the study and the EEG analysis, monitored EEG data reliability, and drafted a significant portion of the manuscript. JCS contributed to the study design, analyzed data, and drafted a significant portion of the manuscript. PJY designed the trial data analysis, analyzed data, created tables, and drafted a significant portion of the manuscript. MLN contributed to the clinical data analysis and interpretation and drafted a significant portion of the manuscript. CR contributed to the clinical data analysis and interpretation and drafted a significant portion of the manuscript. MTW helped conceptualize the study guided the interpretation and drafted a significant portion of the manuscript. MMM helped conceptualize the study, designed and executed the EEG analysis and drafted a significant portion of the manuscript.

Funding This study was supported by National Institutes of Child Health and Human Development, 1 K23 HD074736-01A1; 01/1401/15 to NLM and the Swiss National Science Foundation (Grant 169206 to MMM). The content is solely the responsibility of the authors and does not necessarily represent the official views of the National Institutes of Health.

Data Availability All reasonable requests from qualified scientists for unique research resources (ERP paradigms, protocols and expertise) developed with NIH funds for research purposes will be honored. We will fill requests in a timely manner. We will adhere to the NIH Grant Policy on Sharing of Unique Research Resources including the Sharing of Biomedical Research Resources Principle and Guidelines for Recipients of NIH Grants and Contracts. There is no unique biological information that could be made available to the scientific community. De-identified ERP raw data will be retained on the Abigail Wexner Research Institute at Nationwide Children's Hospital server, and assessment data will be retained in REDCap. These data will be made available to investigators who make specific inquiry for good cause 5 years after the conclusion of the final outcomes.

\section{Compliance with Ethical Standards}

Conflict of interest The authors report no conflict of interest.

Ethical Approval This research was approved by the Vanderbilt University Medical Center Review Board.

Informed Consent Written informed consent was obtained for each subject included in the study. Consent for publication of non-identifiable results is included in the written informed consent.

Open Access This article is licensed under a Creative Commons Attribution 4.0 International License, which permits use, sharing, adaptation, distribution and reproduction in any medium or format, as long as you give appropriate credit to the original author(s) and the source, provide a link to the Creative Commons licence, and indicate if changes were made. The images or other third party material in this article are included in the article's Creative Commons licence, unless indicated otherwise in a credit line to the material. If material is not included in the article's Creative Commons licence and your intended use is not permitted by statutory regulation or exceeds the permitted use, you will need to obtain permission directly from the copyright holder. To view a copy of this licence, visit http://creativecommons.org/licenses/by/4.0/. 


\section{References}

Achenbach TM (2009) The Achenbach System of Empirically Based Assessement (ASEBA): development, findings, theory, and applications. University of Vermont Research Center for Children, Youth, \& Families, Burlington, VT

Achenbach TM, Rescorla LA (2000) Manual for the ASEBA preschool forms \& profiles. University of Vermont, Research Center for Children, Youth, \& Families, Burlington, VT

Adam-Darque A, Pittet MP, Grouiller F, Rihs TA, Leuchter RH, Lazeyras F, Michel CM, Hüppi PS (2020) Neural correlates of voice perception in newborns and the influence of preterm birth. Cereb Cortex 2020:144. https://doi.org/10.1093/cercor/bhaa144

Aspell JE, Lenggenhager B, Blanke O (2012) Multisensory perception and bodily self-consciousness: from out-of-body to inside-body experience. In: Murray MM, Wallace MT (eds) The neural bases of multisensory processes. Frontiers in Neuroscience, Boca Raton, FL

Bahrick LE, Lickliter R (2012) The role of intersensory redundancy in early perceptual, cognitive, and social development. Multisens Dev. https://doi.org/10.1093/acprof:oso/9780199586059.003.0008

Barutchu A, Crewther SG, Fifer J, Shivdasani MN, Innes-Brown H, Toohey S, Danaher J, Paolini AG (2011) The relationship between multisensory integration and IQ in children. Dev Psychol 47:877885. https://doi.org/10.1037/a0021903

Barutchu A, Fifer JM, Shivdasani MN, Crewther SG, Paolini AG (2019a) The interplay between multisensory associative learning and IQ in children. Child Dev 91:620-637. https://doi. org/10.1111/cdev. 13210

Barutchu A, Sahu A, Humphreys GW, Spence C (2019b) Multisensory processing in event-based prospective memory. Acta Physiol (Oxf) 192:23-30. https://doi.org/10.1016/j.actpsy.2018.10.015

Barutchu A, Toohey S, Shivdasani MN, Fifer JM, Crewther SG, Grayden DB, Paolini AG (2019c) Multisensory perception and attention in school-age children. J Exp Child Psychol 180:141155. https://doi.org/10.1016/j.jecp.2018.11.021

Best K, Bogossian F, New K (2018) Language exposure of preterm infants in the neonatal unit: a systematic review. Neonatology 114(3):261-276. https://doi.org/10.1159/000489600

Bhutta AT, Cleves MA, Casey PH, Cradock MM, Anand KJS (2002) Cognitive and behavioral outcomes of school-aged children who were born preterm. JAMA. https://doi.org/10.1001/ jama.288.6.728

Biasiucci A, Franceschiello B, Murray MM (2019) Electroencephalography. Curr Biol 29:R80-R85. https://doi.org/10.1016/j. cub.2018.11.052

Birch HG, Belmont L (1965) Auditory-visual integration, intelligence and reading ability in school. Child Percept Motor Skills 20:295305. https://doi.org/10.2466/pms.1965.20.1.295

Bremner AJ, Lewkowicz DJ, Spence C (2012) Multisensory development, 1st edn. Oxford University Press, Oxford

Brett-Green BA, Miller LJ, Gavin WJ, Davies PL (2008) Multisensory integration in children: a preliminary ERP study. Brain Res 1242:283-290. https://doi.org/10.1016/j.brainres.2008.03.090

Brummelte $S$ et al (2012) Procedural pain and brain development in premature newborns. Ann Neurol 71:385-396. https://doi. org/10.1002/ana.22267

Brunet D, Murray MM, Michel CM (2011) Spatiotemporal analysis of multichannel EEG: CARTOOL. Comput Intel Neurosci 2011:115. https://doi.org/10.1155/2011/813870

Cassiano RGM, Gaspardo CM, Linhares MBM (2016) Prematurity, neonatal health status, and later child behavioral/emotional problems: a systematic review. Infant Ment Health J 37:274-288. https ://doi.org/10.1002/imhj.21563
Denervaud S, Gentaz E, Matusz PJ, Murray MM (2020) Multisensory gains in simple detection predict global cognition in schoolchildren. Sci Rep. https://doi.org/10.1038/s41598-020-58329-4

Dionne-Dostie E, Paquette N, Lassonde M, Gallagher A (2015) Multisensory integration and child neurodevelopment. Brain Sci 5:32-57. https://doi.org/10.3390/brainsci5010032

Dunn W (2002) Infant/toddler sensory profile: user's manual. Psychological Corporation, San Antonio

Eckstein Grunau R (2002) Early pain in preterm infants. Clin Perinatol 29:373-394. https://doi.org/10.1016/s0095-5108(02)00012-x

Foster SM, Verny TR (2007) The development of sensory systems during the prenatal period. J Prenatal Perinatal Psychol Health $21: 271-280$

Foxe JJ, Morocz IA, Murray MM, Higgins BA, Javitt DC, Schroeder CE (2000) Multisensory auditory-somatosensory interactions in early cortical processing revealed by high-density electrical mapping. Cogn Brain Res 10:77-83. https://doi.org/10.1016/s0926 $-6410(00) 00024-0$

Foxe JJ et al (2002) Auditory-somatosensory multisensory processing in auditory association cortex: an fMRI study. J Neurophysiol 88:540-543. https://doi.org/10.1152/jn.2002.88.1.540

Guthrie D, Buchwald JS (1991) Significance testing of difference potentials. Psychophysiology 28(2):240-244. https://doi. org/10.1111/j.1469-8986.1991.tb00417.x

Hack M (2009) Adult outcomes of preterm children. J Dev Behav Pediatr 30:460-470. https://doi.org/10.1097/DBP.0b013e3181ba0fba

Harris PA, Taylor R, Minor BL, Elliot V, Fernandez M, O'Neal L, McLeod L, Delacqua G, Delacqua F, Kirby J, Duda SN, The REDCap Consorium (2019) The REDCap consortium: Building an international community of software platform partners. J Biomed Inform. https://doi.org/10.1016/j.jbi.2019.103208

Hille ETM, Dorrepaal C, Perenboom R, Gravenhorst JB, Brand R, Verloove-Vanhorick SP (2008) Social lifestyle, risk-taking behavior, and psychopathology in young adults born very preterm or with a very low birthweight. J Pediatr 152:793-800.e794. https:// doi.org/10.1016/j.jpeds.2007.11.041

Hofstra MB, Van Der Ende J, Verhulst FC (2018) Adolescents' selfreported problems as predictors of psychopathology in adulthood: 10-year follow-up study. Br J Psychiatry 179:203-209. https://doi. org/10.1192/bjp.179.3.203

Hofstra MB, Van Der Ende JAN, Verhulst FC (2002) Child and adolescent problems predict DSM-IV disorders in adulthood: a 14-year follow-up of a Dutch epidemiological sample. J Am Acad Child Adolesc Psychiatry 41:182-189. https://doi.org/10.1097/00004 583-200202000-00012

Izard V, Sann C, Spelke ES, Streri A (2009) Newborn infants perceive abstract numbers. Proc Natl Acad Sci USA 106:10382-10385. https://doi.org/10.1073/pnas.0812142106

Key AP, Lambert EW, Aschner JL, Maitre NL (2012) Influence of gestational age and postnatal age on speech sound processing in NICU infants. Psychophysiology 49:720-731. https://doi.org/10. 1111/j.1469-8986.2011.01353.x

Lahav A, Skoe E (2014) An acoustic gap between the NICU and womb: a potential risk for compromised neuroplasticity of the auditory system in preterm infants. Front Neurosci 8:381. https://doi. org/10.3389/fnins.2014.00381

Lewkowicz DJ (2014) Early experience and multisensory perceptual narrowing. Dev Psychobiol 56:292-315. https://doi.org/10.1002/ dev.21197

Lewkowicz DJ, Turkewitz G (1980) Cross-modal equivalence in early infancy: auditory-visual intensity matching. Dev Psychol 16:597607. https://doi.org/10.1037/0012-1649.16.6.597

Lickliter R (2011) The integrated development of sensory organization. Clin Perinatol 38:591-603. https://doi.org/10.1016/j. clp.2011.08.007 
Lickliter R, Bahrick LE (2004) Perceptual development and the origins of multisensory responsiveness. The handbook of multisensory processes. MIT Press, Cambridge, pp 643-654

Liszka L, Heiny E, Smith J, Schlaggar BL, Mathur A, Pineda R (2020) Auditory exposure of high-risk infants discharged from the NICU and the impact of social factors. Acta Paediatr. https:// doi.org/10.1111/apa.15209

Machado ACCP, Oliveira SR, Magalhães LC, Miranda DM, Bouzada MCF (2017) Sensory processing during childhood in preterm infants: A systematic review. Rev Paul Pediatr 35(1):92-101. https ://doi.org/10.1590/1984-0462/2017.35.1.00008

Maitre NL, Key AP (2014) Quantitative assessment of cortical auditory-tactile processing in children with disabilities. J Visualiz Exp. https://doi.org/10.3791/51054

Maitre NL, Key AP, Chorna OD, Slaughter JC, Matusz PJ, Wallace MT, Murray MM (2017) The dual nature of early-life experience on somatosensory processing in the human infant brain. Curr Biol 27:1048-1054. https://doi.org/10.1016/j.cub.2017.02.036

Maitre NL, Lambert WE, Aschner JL, Key AP (2013) Cortical speech sound differentiation in the neonatal intensive care unit predicts cognitive and language development in the first years of life. Dev Med Child Neurol 55:834-839. https://doi.org/10.1111/ dmon.12191

Murray MM, Eardley AF, Edginton T, Oyekan R, Smyth E, Matusz PJ (2018) Sensory dominance and multisensory integration as screening tools in aging. Sci Rep. https://doi.org/10.1038/s4159 8-018-27288-2

Murray MM, Lewkowicz DJ, Amedi A, Wallace MT (2016) Multisensory processes: a balancing act across the lifespan. Trends Neurosci 39:567-579. https://doi.org/10.1016/j.tins.2016.05.003

Murray MM, Molholm S, Michel CM, Heslenfeld DJ, Ritter W, Javitt DC, Schroeder CE, Foxe JJ (2005) Grabbing your ear: rapid auditory-somatosensory multisensory interactions in low-level sensory cortices are not constrained by stimulus alignment. Cereb Cortex 15:963-974. https://doi.org/10.1093/cercor/bhh197

Nelson CA (2001) Neural plasticity and human development: the role of early experience in sculpting memory systems. Dev Sci 3:115136. https://doi.org/10.1111/1467-7687.00104

Oller DK, Caskey M, Yoo H, Bene ER, Jhang Y, Lee CC, Bowman DD, Long HL, Buder EH, Vohr B (2019) Preterm and full term infant vocalization and the origin of language. Sci Rep 9(1):14734. https ://doi.org/10.1038/s41598-019-51352-0

Pellegrin M (2020) A deep dive into health insurance coverage in tennessee: trends in coverage, choice, and costs since 2010. The Sycamore Institute. https://www.sycamoreinstitutetn.org/health-insur ance-coverage-trends-tennessee/. Accessed 1 Jun 2020

Perrin F, Pernier J, Bertnard O, Giard MH, Echallier JF (1987) Mapping of scalp potentials by surface spline interpolation. Electroencephalogr Clin Neurophysiol 66:75-81. https://doi. org/10.1016/0013-4694(87)90141-6

Rochat P, Broesch T, Jayne K (2012) Social awareness and early self-recognition. Conscious Cogn 21:1491-1497. https://doi. org/10.1016/j.concog.2012.04.007

Rose SA, Feldman JF, Jankowski JJ, Rossem RV (2008) A cognitive cascade in infancy: pathways from prematurity to later mental development. Intelligence 36:367-378. https://doi.org/10.1016/j. intell.2007.07.003

Rose SA, Feldman JF, Jankowski JJ, Van Rossem R (2012) Information processing from infancy to 11 years: continuities and prediction of IQ. Intelligence 40:445-457. https://doi.org/10.1016/j.intel 1.2012.05.007

Russo N, Foxe JJ, Brandwein AB, Altschuler T, Gomes H, Molholm S (2010) Multisensory processing in children with autism: highdensity electrical mapping of auditory-somatosensory integration. Autism Res 3:253-267. https://doi.org/10.1002/aur.152

Ryckman J, Hilton C, Rogers C, Pineda R (2017) Sensory processing disorder in preterm infants during early childhood and relationships to early neurobehavior. Early Hum Dev 113:18-22. https:// doi.org/10.1016/j.earlhumdev.2017.07.012

Santos J, Pearce SE, Stroustrup A (2015) Impact of hospital-based environmental exposures on neurodevelopmental outcomes of preterm infants. Curr Opin Pediatr 27:254-260. https://doi. org/10.1097/mop.0000000000000190

Sheridan MA, Nelson CA (2009) Neurobiology of fetal and infant development. In: Zeanah $\mathrm{CH}$ (ed) Handbook of infant mental health. Guilford Press, New York, pp 40-58

Sperdin HF (2009) Early, low-level auditory-somatosensory multisensory interactions impact reaction time speed. Front Integr Neurosci. https://doi.org/10.3389/neuro.07.002.2009

Spittle AJ et al (2009) Early emergence of behavior and social-emotional problems in very preterm infants. J Am Acad Child Adolesc Psychiatry 48:909-918. https://doi.org/10.1097/CHI.0b013e3181 af8235

Stephen JM, Romero L, Zhang T, Okada Y (2007) Auditory and somatosensory integration in infants. Int Congr Ser 1300:107-110. https://doi.org/10.1016/j.ics.2007.01.041

Stevens KN, Blumstein SE (1978) Invariant cues for place of articulation in stop consonants. J Acoust Soc Am 64:1358-1368. https:// doi.org/10.1121/1.382102

Thomas RL, Misra R, Akkunt E, Ho C, Spence C, Bremner AJ (2018) Sensitivity to auditory-tactile colocation in early infancy. Dev Sci. https://doi.org/10.1111/desc. 12597

Thompson JL, Nelson AJ (2000) The place of Neandertals in the evolution of hominid patterns of growth and development. J Hum Evol 38:475-495. https://doi.org/10.1006/jhev.1999.0364

Tivadar RI, Murray MM (2018) A primer on electroencephalography and event-related potentials for organizational neuroscience. Organiz Res Methods 22:69-94. https://doi.org/10.1177/10944 28118804657

Turpin H, Urben S, Ansermet F, Borghini A, Murray MM, Müller-Nix C (2019) The interplay between prematurity, maternal stress and children's intelligence quotient at age 11: a longitudinal study. Sci Rep. https://doi.org/10.1038/s41598-018-36465-2

Wallace MT, Stein BE (2007) Early experience determines how the senses will interact. J Neurophysiol 97:921-926. https://doi. org/10.1152/jn.00497.2006

Webb AR, Heller HT, Benson CB, Lahav A (2015) Mother's voice and heartbeat sounds elicit auditory plasticity in the human brain before full gestation. Proc Natl Acad Sci USA 112(10):31523157. https://doi.org/10.1073/pnas.1414924112

Publisher's Note Springer Nature remains neutral with regard to jurisdictional claims in published maps and institutional affiliations. 


\section{Affiliations}

\section{Nathalie L. Maitre ${ }^{1,2,12}$ (D) Alexandra P. Key ${ }^{2,3} \cdot$ James C. Slaughter $^{4} \cdot$ Paul J. Yoder $^{5}$ (D) Mary Lauren Neel ${ }^{1}$ (D) Céline Richard ${ }^{1} \cdot$ Mark T. Wallace $^{2,3,6,7,8} \cdot$ Micah M. Murray $^{2,9,10,11}$ (D)}

1 Center for Perinatal Research at the Abigail Wexner Research Institute, Nationwide Children's Hospital, Columbus, OH, USA

2 Department of Hearing and Speech Sciences, Vanderbilt University Medical Center, Nashville, TN, USA

3 Vanderbilt Kennedy Center, Vanderbilt University Medical Center, Nashville, TN, USA

4 Department of Biostatistics, Vanderbilt University Medical Center, Nashville, TN, USA

5 Department of Special Education, Peabody College of Education and Human Development, Vanderbilt University, Nashville, TN, USA

6 Departments of Psychology and Pharmacology, Vanderbilt University, Nashville, TN, USA
7 Department of Psychiatry and Behavioral Sciences, Vanderbilt University Medical Center, Nashville, TN, USA

8 Vanderbilt Brain Institute, Vanderbilt University, Nashville, TN, USA

9 The Laboratory for Investigative Neurophysiology (The LINE), Department of Radiology, University Hospital Center and University of Lausanne, Lausanne, Switzerland

10 Sensory, Perceptual, and Cognitive Neuroscience Section, Center for Biomedical Imaging (CIBM) of Lausanne, Lausanne, Switzerland

11 Department of Ophthalmology, Fondation Asile des aveugles and University of Lausanne, Lausanne, Switzerland

12 Department of Pediatrics, Nationwide Children's Hospital, 700 Children's Way, Columbus, OH 43205, USA 